\title{
Antiproton-proton annihilation at rest into
}

\author{
$\mathrm{K}^{+} \mathrm{K}^{-} \pi^{0}$
}

\section{Crystal Barrel Collaboration}

A. Abele ${ }^{\text {h }}$, J. Adomeit ${ }^{g}$, C. Amsler ${ }^{\circ}$, C.A. Baker ${ }^{\mathrm{e}}$, B.M. Barnett ${ }^{\text {c,1 }}$, C.J. Batty ${ }^{\mathrm{e}}$, M. Benayoun ${ }^{1}$, A. Berdoz ${ }^{\mathrm{m}}$, S. Bischoff ${ }^{h}$, P. Blüm ${ }^{\text {h }}$, K. Braune ${ }^{\text {k }}$, D.V. Bugg ${ }^{i}$, T. Case $^{\text {a }}$, O. Cramer ${ }^{\mathrm{k}}$, K.M. Crowe ${ }^{\mathrm{a}}$, T. Degener ${ }^{\mathrm{b}}$, M. Doser ${ }^{\mathrm{f}}$, W. Dünnweber ${ }^{\mathrm{k}}$, D. Engelhardt ${ }^{\mathrm{h}}$, M.A. Faessler ${ }^{\mathrm{k}}$, P. Giarritta ${ }^{\circ}$, R.P. Haddock ${ }^{j}$, F.H. Heinsius ${ }^{a, 3}$, M. Heinzelmann ${ }^{\circ}$, A. Herbstrith ${ }^{\mathrm{h}}$, M. Herz ${ }^{\mathrm{c}}$, N.P. Hessey ${ }^{\mathrm{k}}$, P. Hidas ${ }^{\mathrm{d}}$, C. Hodd ${ }^{\mathrm{i}}$, C. Holtzhaußen ${ }^{\mathrm{h}}$, D. Jamnik ${ }^{\mathrm{k}, 4}$, H. Kalinowsky ${ }^{c}$, P. Kammel ${ }^{\text {a }}$, J. Kisiel ${ }^{\text {f, }}$, E. Klempt ${ }^{c}$, H. Koch ${ }^{b}$, M. Kunze ${ }^{\text {b }}$, U. Kurilla ${ }^{\text {b }}$, M. Lakata ${ }^{a}$, R. Landua ${ }^{\mathrm{f}}$, H. Matthäy ${ }^{\mathrm{b}}$, R. McCrady ${ }^{\mathrm{m}}$, J. Meier ${ }^{\mathrm{g}}$, C.A. Meyer ${ }^{\mathrm{m}}$, R. Ouared ${ }^{g}$, K. Peters ${ }^{b}$, B. Pick ${ }^{c}$, M. Ratajczak ${ }^{b}$, C. Regenfus ${ }^{\circ}$, W. Roethel ${ }^{k}$, R. Seibert ${ }^{g}$, S. Spanier ${ }^{\circ}$, H. Stöck ${ }^{b}$, C. Straßburger ${ }^{c}$, U. Strohbusch ${ }^{\mathrm{g}}$, M. Suffert ${ }^{\mathrm{n}}$, J.S. Suh ${ }^{\mathrm{c}}$, U. Thoma ${ }^{\mathrm{c}}$, M. Tischhäuser ${ }^{\mathrm{h}}$, I. Uman ${ }^{\mathrm{k}}$, C. Völcker ${ }^{\mathrm{k}}$, S. Wallis-Plachner ${ }^{\mathrm{k}}$, D. Walther ${ }^{\mathrm{k}, 6}$, U. Wiedner ${ }^{\mathrm{k}}$, K. Wittmack ${ }^{\mathrm{c}}$, B.S. Zou ${ }^{\mathrm{i}}$

${ }^{\text {a }}$ University of California, LBNL, Berkeley, CA 94720, USA

${ }^{\mathrm{b}}$ Universität Bochum, D-44780 Bochum, FRG

${ }^{\mathrm{c}}$ Universität Bonn, D-53115 Bonn, FRG

${ }^{\mathrm{d}}$ Academy of Science, H-1525 Budapest, Hungary

${ }^{\mathrm{e}}$ Rutherford Appleton Laboratory, Chilton, Didcot OX11 0QX, UK

${ }^{\mathrm{f}} \mathrm{CERN}, \mathrm{CH}-1211$ Geneva 4, Switzerland

${ }^{\mathrm{g}}$ Universität Hamburg, D-22761 Hamburg, FRG

${ }^{\mathrm{h}}$ Universität Karlsruhe, D-76021 Karlsruhe, FRG

${ }^{\mathrm{i}}$ Queen Mary and Westfield College, London E1 4NS, UK

${ }^{\mathrm{j}}$ University of California, Los Angeles, CA 90024, USA

${ }^{\mathrm{k}}$ Universität München, D-80333 München, FRG

${ }^{1}$ LPNHE Paris VI, VII, F-75252 Paris, France 
${ }^{\mathrm{m}}$ Carnegie Mellon University, Pittsburgh, PA 15213, USA

${ }^{\mathrm{n}}$ Centre de Recherches Nucléaires,F-67037 Strasbourg, France

${ }^{\circ}$ Universität Zürich, CH-8057 Zürich, Switzerland

\begin{abstract}
The annihilation channel $\overline{\mathrm{p}} \mathrm{p} \rightarrow \mathrm{K}^{+} \mathrm{K}^{-} \pi^{0}$ in liquid hydrogen at rest has been studied with the Crystal Barrel detector at LEAR. The measured branching ratio is: $\mathrm{BR}\left(\overline{\mathrm{p}} \mathrm{p} \rightarrow \mathrm{K}^{+} \mathrm{K}^{-} \pi^{0}\right)=(2.37 \pm 0.15) \times 10^{-3}$. A partial wave analysis shows that this reaction is dominated by the $\pi(\mathrm{KK})_{\mathrm{S}}, \mathrm{KK}^{*}(892)$ and $\pi \phi(1020)$ intermediate states. Weak signals are observed for $\pi \mathrm{a}_{2}(1320), \pi \mathrm{f}_{2}(1270), \pi \mathrm{f}_{2}^{\prime}(1525)$ and $\mathrm{K}(\mathrm{K} \pi)_{\mathrm{S}}$. A satisfactory description of the Dalitz plot requires the introduction of $\pi(\mathrm{KK})_{\mathrm{P}}$ intermediate states with at least one pole.
\end{abstract}

The work described in the present letter continues our systematic study of channels where $\overline{\mathrm{p}}$ annihilates in liquid hydrogen at rest into a 3-body final state containing a $\mathrm{KK}$ pair. In three previous publications [1-3], we have reported on investigations of the channels

$$
\begin{aligned}
& \overline{\mathrm{p}} \mathrm{p} \rightarrow \mathrm{K}_{\mathrm{L}} \mathrm{K}_{\mathrm{S}} \pi^{0} \\
& \overline{\mathrm{p}} \mathrm{p} \rightarrow \mathrm{K}_{\mathrm{L}} \mathrm{K}_{\mathrm{L}} \pi^{0} \\
& \overline{\mathrm{p}} \mathrm{p} \rightarrow \mathrm{K}_{\mathrm{L}} \mathrm{K}^{ \pm} \pi^{\mp}
\end{aligned}
$$

The first two reactions are complementary in that they select opposite $C$ parity eigenstates. Neglecting $C P$-violation in the neutral $\mathrm{KK}$ system, $\mathrm{K}_{\mathrm{L}}$ and $\mathrm{K}_{\mathrm{S}}$ are $C$-parity eigenstates with $C=+1$ and -1 , respectively. Thus, the $C$-parities of the initial $\bar{p}$ p state and of the produced $\mathrm{KK}$ pair are odd for $\mathrm{K}_{\mathrm{L}} \mathrm{K}_{\mathrm{S}} \pi^{0}$ and even for $\mathrm{K}_{\mathrm{L}} \mathrm{K}_{\mathrm{L}} \pi^{0}$.

The partial wave analysis showed that channel (1) is of a striking simplicity: The final state is produced dominantly via two intermediate two-body states, $\mathrm{K}^{*}(892) \overline{\mathrm{K}}$ and $\phi(1020) \pi^{0}$, from the initial $J^{P C}=1^{--}$state of protonium, assuming that only S-wave annihilation contributes. Thus, with only

\footnotetext{
1 Now at University of Mainz, Mainz, Germany

2 Now at Cornell University, Ithaca, USA

3 Now at University of Freiburg, Freiburg,Germany

4 University of Ljubljana, Ljubljana, Slovenia

5 University of Silesia, Katowice, Poland

6 Now at University of Bonn, Bonn, Germany

7 This work is part of the PhD thesis of C.Völcker
} 
two, almost non-interfering amplitudes a satisfactory description of the Dalitz plot containing 2834 events was obtained.

Channel (2) revealed a much more complex interference pattern of amplitudes, with $\mathrm{K}^{*}(892) \mathrm{K}, \mathrm{K}_{0}^{*}(1430) \mathrm{K}, \mathrm{a}_{2}(1320) \pi, \mathrm{f}_{2}(1270) \pi, \mathrm{f}_{2}^{\prime}(1525) \pi$ and a complicated $\pi(\mathrm{KK})_{\mathrm{S}}$ structure as intermediate states, where $(\mathrm{KK})_{\mathrm{S}}$ is a short hand for the $(\mathrm{KK})$-S-wave.$(\mathrm{KK})_{\mathrm{S}}$ may contain $\mathrm{a}_{0}(980), \mathrm{f}_{0}(980), \mathrm{a}_{0}(1450), \mathrm{f}_{0}(1370)$ and $\mathrm{f}_{0}(1500)$. The main results of the analysis of channel (2), based on 37358 events, are the decay branching ratio of $\mathrm{f}_{0}(1500)$ into $\mathrm{KK}$ and the production branching ratio of $\bar{p} p \rightarrow f_{2}^{\prime}(1525) \pi^{0}$.

In channel (3) only $\mathrm{I}=1 \mathrm{~K} \overline{\mathrm{K}}$ resonances are produced, so one can study the $\mathrm{KK}$ decay mode of scalar resonances, in particular the isovectors $\mathrm{a}_{0}(980)$ and $\mathrm{a}_{0}(1450)$, which also fixes the $\mathrm{K} \overline{\mathrm{K}}$ coupling of the isoscalar $\mathrm{f}_{0}(1500)$.

The channel studied in the present work [4] $\overline{\mathrm{p}} \mathrm{p} \rightarrow \mathrm{K}^{+} \mathrm{K}^{-} \pi^{0}$ allows both $C$ parities for the initial $\bar{p} p$ state and the produced $\mathrm{K} \bar{K}$ pair. It is complementary to the channels (1) and (2) in that the experimental techniques to select and measure the final state $\mathrm{K}^{+} \mathrm{K}^{-} \pi^{0}$ are different and that the amplitudes interfere in a different way than those of channels (1) and (2) - the latter feature will be fully exploited in a forthcoming coupled channel analysis of four different $\mathrm{K} \overline{\mathrm{K}} \pi$ final states $\left(\mathrm{K}^{+} \mathrm{K}^{-} \pi, \mathrm{K}_{\mathrm{L}} \mathrm{K}_{\mathrm{L}} \pi^{0}, \mathrm{~K}_{\mathrm{L}} \mathrm{K}_{\mathrm{S}} \pi^{0}\right.$ and $\left.\mathrm{K}^{ \pm} \mathrm{K}_{\mathrm{L}} \pi^{\mp}\right)$. The channel $\mathrm{K}^{+} \mathrm{K}^{-} \pi^{0}$ has so far only been partly analysed by earlier experiments; a complete analysis of the Dalitz plot was hindered by the presence of a strong background from $\pi^{+} \pi^{-} \pi^{0}$, so that only the branching ratio of $\overline{\mathrm{p}} \mathrm{p} \rightarrow \phi(1020) \pi^{0}$ with $\phi(1020) \rightarrow \mathrm{K}^{+} \mathrm{K}^{-}$was extracted [5-7], and, in a rescent publication by the OBELIX-Collaboration [7] also the branching ratio for $\overline{\mathrm{p}} \mathrm{p} \rightarrow \mathrm{f}_{2}^{\prime}(1525) \pi^{0}$ was determined.

This investigation of $\overline{\mathrm{p}} \mathrm{p} \rightarrow \mathrm{K}^{+} \mathrm{K}^{-} \pi^{0}$ is based on a sample of 13.7 million $\overline{\mathrm{p}} \mathrm{p}$ annihilation events measured with the Crystal Barrel detector at the Low Energy Antiproton Ring (LEAR) at CERN. Antiprotons with a momentum of $200 \mathrm{MeV} / \mathrm{c}$ were stopped in a liquid hydrogen target. The target was surrounded by two cylindrical proportional wire chambers (PWC), which were in turn surrounded by a cylindrical jet drift chamber (JDC) with 22 layers and 30 azimuthal sectors for layers 6 to 22 (only 15 azimuthal sectors for layers 1 to 5), to detect charged particles. This inner tracking detector was situated in a barrel-shaped calorimeter consisting of $1380 \mathrm{CsI}(\mathrm{Tl})$ crystals with photodiode readout. The whole detector was located in a solenoid providing a homogeneous field of $1.5 \mathrm{~T}$ parallel to the incident antiproton beam. A detailed description of the apparatus can be found in Ref. [8]. In 1994/1995 the original JDC was replaced by a second JDC, similar in construction apart from minor changes but with superior mechanical precision and reliability. (The number of layers and sectors given above refer to the new JDC). 
13.7 million annihilation events for $\bar{p}$ stopped in liquid hydrogen were taken with a 2-prong-trigger which required a signal in the beam telescope indicating an incoming $\bar{p}$, two clusters in each of the PWCs, and two hits in layers 19 and 20 of the JDC. From this triggered data sample the desired final state was selected by applying cuts to be described below. In addition, $1.6 \times 10^{6}$ minimum bias events, where the only trigger requirement was an incoming $\bar{p}$, were used and submitted to the same cuts in order to determine the branching ratio for $\overline{\mathrm{p}} \mathrm{p} \rightarrow \mathrm{K}^{+} \mathrm{K}^{-} \pi^{0}$ and the acceptance of the 2-prong trigger, with a minimal input from Monte Carlo simulation. The data were selected by imposing the following cuts:

(1) Two long charged tracks were required. Both tracks must begin within the inner three layers (of the JDC) and leave the chamber in one of the 3 outer layers. Moreover, the $\chi^{2} /$ dof of the helix fit to either track must not exceed a value of 1.2.

(2) Two unambiguous particle energy deposits ('PEDs') in the crystal calorimeter were required, to be identified with two showers induced by $\gamma$ rays and which could not be associated with the interaction of one of the charged tracks, and where the central crystal had an energy of at least $13 \mathrm{MeV}$.

(3) An upper bound for the momentum balance was imposed $p_{t o t} c \leq 2450 \mathrm{MeV}-$ $1.2 \cdot E_{t o t}$, where $E_{t o t}$ is the total energy under the hypothesis that the two charged tracks are kaons. Figure 1 shows the cutting line in a two dimensional scattergram $p_{\text {tot }}$ versus $E_{\text {tot }}$ where the desired events $\left(\mathrm{K}^{+} \mathrm{K}^{-} \gamma \gamma\right)$ are seen as a rather distinguished density maximum, separate from others. At this level, the background under this density maximum is about $10 \%$.

By the cuts 1) to 3) the original sample of 13.7 Million events is reduced to about 175000 . As the final step in event selection, a five constraint kinematic fit was applied for the hypothesis $\bar{p} \mathrm{p} \rightarrow \mathrm{K}^{+} \mathrm{K}^{-} \pi^{0}$, with 4 constraints from energy and momentum conservation and one for the invariant mass of the two photons. A confidence level of larger than 0.1 was required for the good hypothesis and a confidence level smaller than 0.01 for the alternative hypothesis of $\pi^{+} \pi^{-} \pi^{0}$, the most important background channel. These cuts left 27158 events for the final analysis.

The unbinned Dalitz plot and its projections are shown in Figure 2. The presence of the $\phi(1020) \pi^{0}$ and the $\mathrm{K}^{*}(892) \mathrm{K}$ intermediate states can be immediately recognized in the Dalitz plot and the projections. The $\phi \rightarrow \mathrm{K}^{+} \mathrm{K}^{-}$is seen as a diagonal band on the upper right edge of the Dalitz plot and the $\mathrm{K}^{*} \mathrm{~s}$ are seen as vertical and horizontal bands, forming a cross. The upper right and the lower left quadrants relative to this cross are filled with intensity, due to constructive interference with the $(\mathrm{KK}) \mathrm{S}$ - and D-waves. This interference pattern differs in a remarkable way from those seen in channels (1) and (2). 
As possible sources of background, channels were considered which have the same topology ( 2 tracks, two gammas) as $\mathrm{K}^{+} \mathrm{K}^{-} \pi^{0}$, i.e. the following channels
a) $\overline{\mathrm{p}} \mathrm{p} \rightarrow \mathrm{K}_{\mathrm{L}} \mathrm{K}^{ \pm} \pi^{\mp} \pi^{0}$
2 tracks, 2 PEDs
$\mathrm{BR}=(4.69 \pm 0.55) \times 10^{-3}[9]$
b) $\overline{\mathrm{p}} \mathrm{p} \rightarrow \mathrm{K}_{\mathrm{L}} \mathrm{K}_{\mathrm{S}} \pi^{0}, \mathrm{~K}_{\mathrm{S}} \rightarrow \pi^{+} \pi^{-}$
2 tracks, 2 PEDs $\quad \mathrm{BR}=(0.47 \pm 0.10) \times 10^{-3}[10]$
c) $\overline{\mathrm{p}} \mathrm{p} \rightarrow \pi^{+} \pi^{-} \pi^{0}$
2 tracks, 2 PEDs $\quad \mathrm{BR}=(6.6 \pm 0.8) \times 10^{-2}[11]$
d) $\overline{\mathrm{p}} \mathrm{p} \rightarrow \pi^{+} \pi^{-} \omega, \omega \rightarrow \pi^{0} \gamma$
2 tracks, 3 PEDs
$\mathrm{BR}=(5.6 \pm 0.5) \times 10^{-3}[12]$

The first two channels are produced with branching ratios of the same order as the channel $\mathrm{K}^{+} \mathrm{K}^{-} \pi^{0}$, the last two have branching ratios an order of magnitude larger. These channels were simulated with the Crystal Barrel event simulation program CBGEANT which is based on the GEANT program package [13]. In all four cases a negligible number of events passes the selection criteria. We conclude that the Dalitz plot contains less than $0.1 \%$ of background events. For example, for each of the two strong channels 300000 Monte Carlo events were generated and only one event per channel passed, while the acceptance for the $\mathrm{K}^{+} \mathrm{K}^{-} \pi^{0}$ channel is $10.0 \%$.

The Dalitz plot of Figure 2 is not yet acceptance corrected. The correction of the data was performed in two steps. Using the CBGEANT program, a sample of 2.75 million phase space distributed events for $\bar{p} \mathrm{p} \rightarrow \mathrm{K}^{+} \mathrm{K}^{-} \pi^{0}$ with $\pi^{0} \rightarrow \gamma \gamma$ were generated and submitted to the same cuts as the real events. The Dalitz plot was divided into $50 \times 50$ bins with a size of $0.036 \times 0.036\left(\mathrm{GeV}^{2} / c^{4}\right)^{2}$. For every bin of the Dalitz plot an acceptance correction factor was obtained. After this standard procedure, an additional acceptance correction was applied to account for the known fact that the simulation package FLUKA of GEANT does not sufficiently well describe hadronic interactions at low energy. In particular, FLUKA does not distinguish between $\mathrm{K}^{+}$and $\mathrm{K}^{-}$interactions at momenta below $300 \mathrm{MeV} / \mathrm{c}$, neglecting the important process of $\mathrm{K}^{-}$absorption by a nucleus. The properties of energy deposits, such as PED multiplicity per hadron interaction, have an impact on the acceptance via the topology cut which requires a specific number of unambiguous PEDs.

Therefore as a first step, momentum dependent correction factors for $\mathrm{K}^{+}$interactions were determined, comparing simulated with real $\mathrm{K}^{+}$interactions. For the latter, out of $1.3 \times 10^{6}$ minimum bias events about $10^{4}$ events were selected with a $\mathrm{K}^{+}$track, well identified by the $\mathrm{dE} / \mathrm{dx}$ measurement in the JDC. (Note, by the way, that no $\mathrm{dE} / \mathrm{dx}$ information was used for the selection of the $\mathrm{K}^{+} \mathrm{K}^{-} \pi^{0}$ channel.) Comparing the number of events where no track-associated PED was found for the real and the simulated events led to the momentum dependent acceptance correction function for the $\mathrm{K}^{+}$. The function is unity for $p_{K^{+}}>200 \mathrm{MeV} / c$ and rises up to 1.2 for decreasing $\mathrm{K}^{+}$momentum. 
Secondly, after the application of the $\mathrm{K}^{+}$correction factors, a momentum dependent correction accounting for the low momentum $\mathrm{K}^{-}$interactions was obtained from the Dalitz plot asymmetry with respect to interchanging $\mathrm{K}^{+}$ and $\mathrm{K}^{-}$, assuming that the $\mathrm{K}^{+}$acceptance was, at this point, correctly described. The function by which each bin of the Dalitz plot has been multiplied is unity for $p_{K^{-}}>400 \mathrm{MeV} / c$ and decreases to 0.7 with decreasing $\mathrm{K}^{-}$momentum. The effect of all acceptance corrections is illustrated in Figure 2, by superimposing the acceptance-corrected projections, scaled down by a factor of 10 .

We begin with the determination of the branching ratio although this requires information from the complete partial wave analysis which is described later in the paper. From the $1.6 \times 10^{6}$ minimum bias events, $387 \mathrm{~K}^{+} \mathrm{K}^{-} \pi^{0}$ events were selected, applying the same cuts as for the 2-prong triggered sample. Instead of using the average reconstruction efficiency of $10.0 \%$ obtained from phase space distributed Monte Carlo events (see above), the correctly averaged reconstruction efficiency was obtained by weighting each bin of the Dalitz plot with the theoretical intensity, as determined by the partial wave analysis of the experimental Dalitz plot. The weighted average acceptance is $(10.90 \pm 0.15) \%$. Subtracting $(5.7 \pm 1.1) \%$ of annihilations which occur in flight - this number was obtained in an earlier analysis [14]- we obtain a branching ratio

$$
B R\left(\overline{\mathrm{p}} \mathrm{p} \rightarrow \mathrm{K}^{+} \mathrm{K}^{-} \pi^{0}\right)=(2.37 \pm 0.15) \times 10^{-3} .
$$

The result agrees well with that of an independent analysis [15] of the same data:

$$
B R\left(\overline{\mathrm{p}} \mathrm{p} \rightarrow \mathrm{K}^{+} \mathrm{K}^{-} \pi^{0}\right)=(2.55 \pm 0.15) \times 10^{-3} .
$$

and with the value obtained by the OBELIX Collaboration [16] :

$$
B R\left(\overline{\mathrm{p}} \mathrm{p} \rightarrow \mathrm{K}^{+} \mathrm{K}^{-} \pi^{0}\right)=(2.37 \pm 0.16) \times 10^{-3} .
$$

The main contribution to the error is statistical, but the error also includes the uncertainties of the acceptance and background estimates.

The partial wave analysis assumed intermediate states with resonances between any pair of final state hadrons. Possible resonances are those listed in the introduction for channels (1) and (2). The data require in addition the inclusion of higher mass $\rho$, resp. $\omega$ resonances as will be shown below.

The differential intensity $I$ of events entering the Dalitzplot was parametrized 
by

$$
I(\vec{p}, \vec{q})=\sum_{J^{P C}}\left|\sum_{l, L, i, I} A_{l, L, J^{P C}, i, I}(\vec{p}, \vec{q})\right|^{2}
$$

where the sum extends over the two initial S-states of protonium with $J^{P C}=0^{-+}$ and $1^{--}$, over the angular momenta $l, L$ and over the isospins $i$ and $I$ of intermediate resonance and initial state, respectively. Small $l$ is the angular momentum of the resonance decay (equal to its spin in the present case of decay into pseudoscalars) and $L$ is the angular momentum between resonance and the third (spectator) particle. $\vec{p}$ is the momentum of the third particle recoiling against the resonance, in the $\bar{p} p$ CM-system, and $\vec{q}$ is the momentum of one of the resonance products, in the resonance CM-system. The amplitudes $A$ are given by the product

$$
A_{l, L, J}^{P C}, i, I(\vec{p}, \vec{q})=Z_{l, L, J}(\vec{p}, \vec{q}) \cdot D_{L}(|\vec{p}|) \cdot \tau_{i, I, C} \cdot F_{l}(|\vec{p}|,|\vec{q}|),
$$

where $Z$ describes the angular dependence. It was parametrized in the tensor formalism of Zemach [17]; $D_{L}$ are the centrifugal barrier factors according to Blatt-Weisskopf [18], $\tau$ are Clebsch-Gordan coefficients connecting various terms of the properly symmetrized total amplitude. $F_{l}$ are dynamical functions; they are described by Breit-Wigner functions, the Flatté formula or by a $\hat{K}$-matrix and a $\hat{P}$-vector. Explicit expressions for the phase space factor $\rho$, the matrix $\hat{K}$ and the vector $\hat{P}$ can be found in Ref. [2]. They contain four free parameters for each intermediate resonance, the complex production strength $\beta_{\alpha}=\left|\beta_{\alpha}\right| \cdot \exp i \phi_{\alpha}$, the $\hat{K}$-matrix pole $m_{\alpha}$ and the total width $\Gamma_{\alpha}$ for each intermediate resonance. These parameters are determined by minimizing a $\chi^{2}$ function (eq. 28.9 in [19]) which compares the experimental and theoretical contents of each bin in the $\mathrm{K}^{+} \mathrm{K}^{-} \pi^{0}$ Dalitz plot, using the program package MINUIT [20]. The results, quoted in Table 1, are the fitted numbers $m_{\alpha}, \Gamma_{\alpha},\left|\beta_{\alpha}\right|$ and $\phi_{\alpha}$. The phase $\phi_{\alpha}$ is always given relative to the phase of the $\mathrm{K}^{*}(892) \mathrm{K}$ amplitude. The contribution of different intermediate states, given as a rate in \%, is extracted by integrating the modulus square of the desired amplitude over the available phase space, normalized by the integral total intensity $I(\vec{p}, \vec{q})$ over the Dalitz plot. The rates do not add up to $100 \%$ since interference terms have been omitted from the table. However, the subtotals for the two initial $\bar{p}$ p states include all interferences within each initial state and therefore yield together $100 \%$.

In a first attempt to fit the $\mathrm{K}^{+} \mathrm{K}^{-} \pi^{0}$ Dalitz plot, only amplitudes corresponding to well established $\mathrm{KK}$ and $\mathrm{K} \pi$ resonances were considered. Since many known resonances are expected to contribute, the number of fit parameters would be too large, if all masses and widths would have been left free. Therefore some widths and masses of well known resonances were fixed in accor- 
dance with values of the PDG [19]. However mass and width of $\mathrm{K}^{*}(892)$ and $\phi(1020)$ were left free, since even a small discrepancy due to our experimental mass resolution would have significantly deteriorated the overall fit due to the dominance of these relatively narrow resonances. The following intermediate 2-body reactions represent the basic hypothesis.

A) Initial $\bar{p}$ p state $J^{P C}=0^{-+} \rightarrow$

(1) $\mathrm{a}_{0} / \mathrm{f}_{0}(980) \pi^{0}$; these two resonances cannot be distinguished and are included as one state, parametrized with a Flatté formula.

(2) $\mathrm{a}_{2}(1320) \pi^{0}$, fixed mass and width taken from PDG.

(3) $\mathrm{K}^{*}(892) \mathrm{K}$, mass and width left free in the fits.

(4) $(\mathrm{K} \pi)_{\mathrm{S}} \mathrm{K}$, with the parametrization taken from Ref. [2] for $(\mathrm{K} \pi)_{\mathrm{S}}$, the $(\mathrm{K} \pi)-$ S-wave.

(5) $(\mathrm{KK})_{\mathrm{S}} \pi^{0}$, the $(\mathrm{KK})$-S-wave has two $K$-matrix poles at $m_{1}=1361 \mathrm{MeV} / c^{2}$, $\Gamma_{1}=264 \mathrm{MeV} / c^{2}$ and $m_{2}=1571 \mathrm{MeV} / c^{2}, \Gamma_{2}=168 \mathrm{MeV} / c^{2}$. The values were taken from Ref. [21] and are fixed in the fits. There is no pole for the $\mathrm{f}_{0}(980)$, see 1 . above.

(6) $\mathrm{f}_{2}(1270) \pi^{0}$, fixed mass and width taken from PDG.

(7) $\mathrm{f}_{2}^{\prime}(1525) \pi^{0}$, fixed mass and width taken from PDG.

B) Initial pp state $J^{P C}=1^{--} \rightarrow$

(1) $\mathrm{K}^{*}(892) \mathrm{K}$, mass and width left free in the fits.

(2) $\phi(1020) \pi^{0}$, mass and width left free in the fits.

The results of this fit are shown in Fig. 3. At first sight, the fit describes the data rather well with a $\chi^{2} /$ dof of 1.50 . The fitted masses and widths also agree well with the accepted values. Whereas the $\mathrm{K}^{ \pm} \pi^{0}$ spectrum is well reproduced, there are significant discrepancies in the $\mathrm{K}^{+} \mathrm{K}^{-}$spectrum at invariant masses squared around 2, 2.4 and $2.7 \mathrm{GeV}^{2} / c^{4}$. Therefore, further amplitudes were added to the basic hypothesis.

The scalar isovector $\mathrm{a}_{0}(1450)$, observed in $\overline{\mathrm{p}} \mathrm{p}$ annihilation into $\pi^{0} \pi^{0} \eta$ and decaying into $\pi^{0} \eta$ [22], respectively $\overline{\mathrm{p}} \mathrm{p} \rightarrow \pi^{0} \pi^{0} \eta^{\prime}, \mathrm{a}_{0}(1450) \rightarrow \pi^{0} \eta^{\prime}$ [23] and in $\mathrm{K}_{\mathrm{L}} \mathrm{K}^{ \pm}[3]$, is also expected to decay to $\mathrm{K}^{+} \mathrm{K}^{-}$. If it is introduced with free parameters, the fits do not converge. Acceptable fits are only obtained with fixed mass, width and production strength for the $\mathrm{a}_{0}(1450)$. A maximum rate of $20 \% \mathrm{a}_{0}(1450)$ is tolerable without changing the quality of the fit and the contributions of the other amplitudes significantly. From a systematic study of these correlations, an upper limit of $\mathrm{a}_{0}(1450)$ production and decay into $\mathrm{K}^{+} \mathrm{K}^{-}$is extracted: $\mathrm{BR}\left(\overline{\mathrm{p}} \mathrm{p} \rightarrow \mathrm{a}_{0}(1450) \pi^{0}\right) \times \mathrm{BR}\left(\mathrm{a}_{0} \rightarrow \mathrm{K}^{+} \mathrm{K}^{-}\right)<(5.4 \pm$ $0.4) \times 10^{-4}$. This upper limit is consistent with the branching ratio measured in [3]: $\mathrm{BR}\left(\overline{\mathrm{p}} \mathrm{p} \rightarrow \mathrm{a}_{0}(1450) \pi^{0}\right)=(8.9 \pm 1.7) \times 10^{-4}$, which leads to $\mathrm{BR}\left(\overline{\mathrm{p}} \mathrm{p} \rightarrow \mathrm{a}_{0}(1450) \pi^{0}\right.$ $\left.\rightarrow \mathrm{K}^{+} \mathrm{K}^{-} \pi^{0}\right)=(8.9 / 6=1.48) \times 10^{-4}$. No evidence was found for an additional scalar resonance above $1600 \mathrm{MeV} / c^{2}$, e.g. the $\mathrm{f}_{0}(1720)$.

An additional tensor resonance around $1700 \mathrm{MeV} / c^{2}$ is rejected by the fit. If a 
single $\mathrm{f}_{2}^{\prime}(1525)$ is assumed with free mass and a starting value of $1700 \mathrm{MeV} / c^{2}$ the mass is pulled down by the fit to $1525 \mathrm{MeV} / c^{2}$, although the contribution of this state is below $1 \%$.

The only remaining possibility to improve the description is the introduction of vector mesons decaying to $\mathrm{K}^{+} \mathrm{K}^{-}$. The available phase space allows contributions from the five known resonances $\omega(1420), \rho(1450), \omega(1600), \phi^{\prime}(1680)$ and $\rho(1700)$. The best fit, which has a $\chi^{2} /$ dof of 1.18 , requires two poles in the $(\mathrm{KK})-\mathrm{P}$-wave, one around $1400 \mathrm{MeV} / c^{2}$ and one near $1700 \mathrm{MeV} / c^{2}$. The introduction of only one of those poles gives a worse result and if more than two poles are allowed, the fits become unstable. However, we attribute a weaker significance to the lower mass pole because of the complex interference of $f_{0}(1370)$ with a possible $a_{0}(1450)$ in this mass region. The lower mass pole may correspond either to $\omega(1420)$ or $\rho(1450)$. For the higher mass pole, there are even three candidates, $\omega(1600), \phi^{\prime}(1680)$ or $\rho(1700)$. The different isospins cannot be distinguished in the $\mathrm{K}^{+} \mathrm{K}^{-} \pi^{0}$ final state. The results of the final fit are listed in Table 1 and the invariant mass distributions are compared in Fig. 4. The Dalitz plot and both projections are well reproduced by the fit.

We are now in the position to calculate the production branching ratios of various intermediate states from the two initial S-wave $\bar{p}$ p states $J^{P C}=0^{-+}$ and $1^{--}$, and to compare them with previous measurements. These branching ratios are obtained by multiplying the relative rate shown in column 3 of Table 1 by the branching ratio $\mathrm{BR}\left(\overline{\mathrm{p}} \mathrm{p} \rightarrow \mathrm{K}^{+} \mathrm{K}^{-} \pi^{0}\right)=(2.37 \pm 0.15) \times 10^{-3}$ and by Clebsch-Gordan coefficients in order to account for all possible charge states of the intermediate $\mathrm{K} \overline{\mathrm{K}}$ or $\mathrm{K} \pi$ resonances. The latter factors are 2 for $\mathrm{f} \pi$ and 6 for $\mathrm{a} \pi, \rho \pi$ and $\mathrm{K}^{*} \mathrm{~K}$. For $\phi \pi$, the PDG value [19] for $B R(\phi \rightarrow \mathrm{KK}) / B R\left(\phi \rightarrow \mathrm{K}^{+} \mathrm{K}^{-}\right)$ $=0.832 / 0.491$ was taken, as for $\mathrm{f}_{2}^{\prime}(1525) \pi\left(B R\left(\mathrm{f}_{2}^{\prime} \rightarrow \mathrm{KK}\right)=0.89\right)$. We do not know the isospin of the observed $(\mathrm{KK})_{P}$ resonances, but assume in the following that they are $\rho$-mesons, hence a factor 6 was applied to calculate the branching ratios.

The resulting branching ratios are listed in Table 2. We have omitted the branching ratio for $\mathrm{a}_{0}(980) \pi$, since the measured contribution of $25.9 \%$ in the Dalitzplot (see Table 1 ) apperently contains more than just the $\mathrm{a}_{0} / \mathrm{f}_{0}(980)$. The fit uses the high mass tail of $\mathrm{a}_{0}(980)$ to account for a flat physical background. The nature of this background may be similar to that observed in the $(\pi \pi)_{S}$ wave.

The majority of branching ratios in Table 2 agree reasonably well with previous measurements. We now discuss likely or possible explanations for some apparent discrepancies.

The branching ratio $\mathrm{BR}\left(\overline{\mathrm{p}} \mathrm{p} \rightarrow \pi \mathrm{a}_{2}\right)$ is larger than most of the previous results. However, the $\mathrm{a}_{2} \rightarrow \mathrm{KK}$ branching ratio is still an open issue. We know that, 
compared with the branching ratio obtained in a previous coupled channel analysis of $\overline{\mathrm{p}} \mathrm{p} \rightarrow \pi \pi \pi, \pi \pi \eta$ and $\eta \eta \pi$, and using the relative decay branching ratio of $\mathrm{a}_{2} \rightarrow \pi \eta$ and $\rightarrow \mathrm{K} \overline{\mathrm{K}}$ from the $\mathrm{PDG}$, the production $\mathrm{BR}$ obtained from $\mathrm{KK} \pi$ final states are in general much too low, by a factor of 2 . The branching ratio of this analysis is somewhat larger than that of the $\mathrm{K}_{\mathrm{L}} \mathrm{K}_{\mathrm{L}} \pi^{0}$ analysis [2].

$\mathrm{BR}\left(\overline{\mathrm{p}} \mathrm{p} \rightarrow \mathrm{K}^{*}(890) \mathrm{K}\right)$ from the initial state $0^{-+}$has been compared with the incoherent sum of the values for the two initial $I$-spins 0 and 1 , as obtained by [24], and it agrees quite well with this sum. This indicates that the phase between the $I=0$ and the $I=1$ amplitude is close to $90^{\circ}$.

$\mathrm{BR}\left(\overline{\mathrm{p}} \mathrm{p} \rightarrow \mathrm{f}_{0}(1370) \pi\right)$ agrees quite well with that obtained in our previous analysis [2], assuming in both cases zero $\mathrm{a}_{0}(1450)$ intensity. However, the values for $\mathrm{f}_{0}(1500)$ disagree by a large factor. This, in turn, is strong support for the need of $\mathrm{a}_{0}(1450)$ since it has been shown in the previous analysis of $\mathrm{K}_{\mathrm{L}} \mathrm{K}_{\mathrm{L}} \pi^{0}$ that the $\mathrm{f}_{0}(1500)$ intensity decreases to about $1 / 2$ of its initial value, when the assumed $\mathrm{a}_{0}(1450)$ intensity is increased from $0 \%$ to $7.5 \%$, whereas in $\mathrm{K}^{+} \mathrm{K}^{-} \pi^{0}$ it increases by $20 \%$. The opposite trends for $\mathrm{K}^{+} \mathrm{K}^{-} \pi^{0}$ and $\mathrm{K}_{\mathrm{L}} \mathrm{K}_{\mathrm{L}} \pi^{0}$ can be explained by the opposite signs of the isospin Clebsch-Gordon coefficients connecting $\mathrm{a}_{0} \pi$ and $\mathrm{f}_{0} \pi$ amplitudes in $\mathrm{K}^{+} \mathrm{K}^{-} \pi^{0}$ and $\mathrm{K}_{\mathrm{L}} \mathrm{K}_{\mathrm{L}} \pi^{0}$.

$\mathrm{BR}(\overline{\mathrm{p}} \mathrm{p} \rightarrow \phi \pi)$ agrees well with previous measurements of Crystal Barrel $[1,25]$ and Obelix [16] and provides support for a strong OZI violation.

We do not, at present, have an explanation for the discrepancy between the values for $\mathrm{f}_{2}^{\prime}(1525)$ as obtained in different analyses.

$\mathrm{BR}\left(\overline{\mathrm{p}} \mathrm{p} \rightarrow \mathrm{K}^{*} \mathrm{~K}\right)$ from initial state $1^{--}$of $\mathrm{K}^{+} \mathrm{K}^{-} \pi^{0}$ seems to disagree with the value from $\mathrm{K}_{\mathrm{L}} \mathrm{K}_{\mathrm{S}} \pi^{0}$; however, a possible explanation of the difference is the following: $K^{*} K$ can be produced from both isospin states, $\mathrm{I}=0$ and $\mathrm{I}=1$. The relative phases between the two amplitudes associated with initial isospin 0 and 1 which add coherently, should be, in general, different. They are given by the common relative production phase, in the case of $\mathrm{K}^{+} \mathrm{K}^{-} \pi^{0}$ and by that same phase plus $180^{\circ}$ in the case of $\mathrm{K}_{\mathrm{L}} \mathrm{K}_{\mathrm{S}} \pi^{0}$ - the phase difference of $180^{\circ}$ is due to isospin Clebsch Gordan coefficients. If $\beta_{0}$ and $\beta_{1}$ denote the moduli of the production strength from $\mathrm{I}=0$ and 1 , respectively, and $\theta$ their relative phase, for the final state $\mathrm{K}^{+} \mathrm{K}^{-} \pi^{0}$ the total amplitude is proportional to $\beta_{0}+\beta_{1} \exp (i \theta)$, whereas it is proportional to $\beta_{0}-\beta_{1} \exp (i \theta)$ for $\mathrm{K}_{\mathrm{L}} \mathrm{K}_{\mathrm{S}} \pi^{0}$. Only for the exceptional case apparently given for the initial $0^{-+}$state - that the relative production phase is $90^{\circ}$ or that one of the two isospin states dominates strongly- does this flip of signs not have an effect. Thus, $B R\left(\bar{p} p \rightarrow K^{*} K\right)$ from initial state $1^{--}$cannot be directly compared with the value obtained from the analysis of the final state $\mathrm{K}_{\mathrm{L}} \mathrm{K}_{\mathrm{S}} \pi^{0}[1]$, whereas $\mathrm{BR}(\overline{\mathrm{p}} \mathrm{p} \rightarrow \phi \pi)$ can be compared, since, the state $\phi \pi$ is produced from a single initial state with $\mathrm{I}=1$.

In summary, most of the branching ratios obtained by this analysis agree 
with previous measurements. Some seeming disagreements can be explained either by a different isospin superposition (the case of $\mathrm{BR}\left(\overline{\mathrm{p}} \mathrm{p} \rightarrow \mathrm{K}^{*} \mathrm{~K}\right)$ ) or by the interference with an additional resonance $\left(a_{0}(1450)\right.$ in case of $\left.f_{0}(1500)\right)$. A new result is the observation of a $(\mathrm{K} \overline{\mathrm{K}})$-P-wave with two effective poles. The lower mass pole may be identified with $\rho(1450)$ or $\omega(1420)$ and the higher mass pole could be either $\omega(1420), \phi^{\prime}(1680)$ or $\rho(1700)$. The decay of $\rho(1700)$ into $\mathrm{KK}$ has already been reported by OBELIX [7], our results add further evidence for the $\mathrm{K} \bar{K}$ decay of $\rho(1450)$ or $\omega(1420)$.

\section{Acknowledgements}

We would like to thank the technical staff of the LEAR machine group and of all the participating institutions for their invaluable contributions to the success of the experiment. We acknowledge financial support from the German Bundesministerium für Bildung, Wissenschaft, Forschung und Technologie, the Schweizerischer Nationalfonds, the British Particle Physics and Astronomy Research Council, the U.S. Department of Energy and the National Science Research Fund Committee of Hungary (contract No. DE-FG03-87ER40323, DE-AC03-76SF00098, DE-FG02-87ER40315 and OTKA F014357). K. M. C., N. D., F. H. H. and R.O. acknowledge support from the Alexander von Humboldt Foundation.

\section{References}

[1] C. Amsler et al., Phys. Lett. B319(1993)373.

[2] A. Abele et al., Phys. Lett. B385(1996)425.

[3] A.Abele et al., Phys. Rev. D57(1998)3860.

[4] C. Völcker, PhD thesis, Universität München 1997.

[5] M. Chiba et al., Phys. Rev. D32(1988)2021, Phys. Lett. B202(1988)447.

[6] J. Reifenröther et al., Phys. Lett. B267(1991)299.

[7] A.Bertin et al., Phys. Lett. B343(1998)180.

[8] E. Aker et al., Nucl. Instr. Methods A321(1992)69.

[9] N. Barash et al., Phys. Rev. B139(1965)1659.

[10] H.P. Dietz, PhD thesis, Universität München 1994.

[11] C. Amsler and F. Myhrer, Ann. Rev. Nucl. Part. Sci. 41(1991)219. 
[12] R. Bizarri et al., Nucl. Phys. B14(1969)169.

[13] GEANT 3.21, CERN program library W5013 (1994).

[14] C. Amsler et al., Z. Phys. C58(1993)175.

[15] R.Seibert, PhD thesis, Universität Hamburg 1997.

[16] A.Alberico et al., Phys. Lett. B438(1998)430.

[17] C. Zemach, Phys. Rev. B133(1964)1201, Phys. Rev. B140(1965)97, Phys. Rev. B140(1965) 109 .

[18] J.M. Blatt and V. Weisskopf, Theoretical Nuclear Physics, Wiley, New York (1952).

[19] Review of Particle Physics, Eur.Phys. J. C 3,1-794(1998).

[20] CERN Program Library Entry D506, MINUIT, Version 92.1 (1992).

[21] S. v.Dombrowski, PhD thesis, Universität Zürich, 1996.

[22] C. Amsler et al., Phys. Lett. B333(1994)277.

[23] A. Abele et al., Phys. Lett. B404(1997)179.

[24] B. Conforto et al., Nucl. Phys. B3(1967)469.

[25] C. Amsler et al., Phys. Lett. B346(1995)363.

[26] S. Prakhov, Workshop on Strangeness in the Nucleon, CERN, Geneva, March 1997. 


\begin{tabular}{|l|c|r|r|}
\hline Intermediate state & $m_{0}, \Gamma_{0}\left[\mathrm{MeV} / c^{2}\right]$ & Rate $[\%]$ & Phase \\
\hline$J^{P C}(\overline{\mathrm{p} p})=0^{-+}$ & & 54.7 & \\
\hline$\pi f_{0} / \mathrm{a}_{0}(980)$ & $(997.6,95.6)$ & $25.9 \pm 3.0$ & $0.38 \pm 0.31$ \\
$\pi \mathrm{a}_{2}(1320)$ & $(1318.1,107)$ & $7.8 \pm 1.0$ & $1.12 \pm 0.24$ \\
$\mathrm{KK}{ }^{*}(892)$ & $893.5 \pm 1.1,54.8 \pm 1.7$ & $10.4 \pm 1.7$ & $(0.0)$ \\
$\mathrm{K}(\mathrm{K} \pi)_{\mathrm{S}}$ & & & \\
pole & $(1342,400)$ & $0.3 \pm 2.0$ & $3.71 \pm 0.31$ \\
scattering & $(\mathrm{a}=2.58 \mathrm{GeV}, \mathrm{b}=1.81 \mathrm{GeV})$ & $2.0 \pm 3.5$ & \\
sum & & $3.7 \pm 6.7$ & \\
$\pi(\mathrm{KK})_{\mathrm{S}}\left(\mathrm{f}_{0}\right)$ & & & \\
pole 1 & $(1361,264)$ & $50.7 \pm 6.3$ & $5.10 \pm 0.20$ \\
pole 2 & $(1571,168)$ & $3.6 \pm 1.0$ & $6.00 \pm 0.26$ \\
sum & & $50.5 \pm 5.9$ & \\
$\pi \mathrm{f}_{2}(1270)$ & $(1275,185)$ & $4.4 \pm 1.0$ & $2.84 \pm 0.26$ \\
$\pi \mathrm{f}_{2}^{\prime}(1525)$ & $1422.8 \pm 6.5,146.5 \pm 10.5$ & $7.4 \pm 1.3$ & $3.91 \pm 0.14$ \\
\hline \hline$J^{P C}(\overline{\mathrm{p} p})=1^{--}$ & & $0.6 \pm 0.3$ & $3.36 \pm 0.23$ \\
\hline $\mathrm{KK}{ }^{*}(892)$ & & 45.3 & \\
$\pi \phi(1020)$ & & $11.8 \pm 0.9$ & $(0.0)$ \\
$\pi(\mathrm{KK})_{\mathrm{P}}$ & & & $2.61 \pm 0.9$ \\
pole 1 & & & $2.87 \pm 0.42$ \\
pole 2 & & & \\
sum & & & \\
\hline
\end{tabular}

Table 1

Results of the best fit with two poles in the $(\mathrm{K} \overline{\mathrm{K}})_{P-\text { wave }}$. Masses and widths given in parentheses were not left free in the fit. Those determined by the fit are the $K$-matrix parameters. See the text for the normalization of rates. 


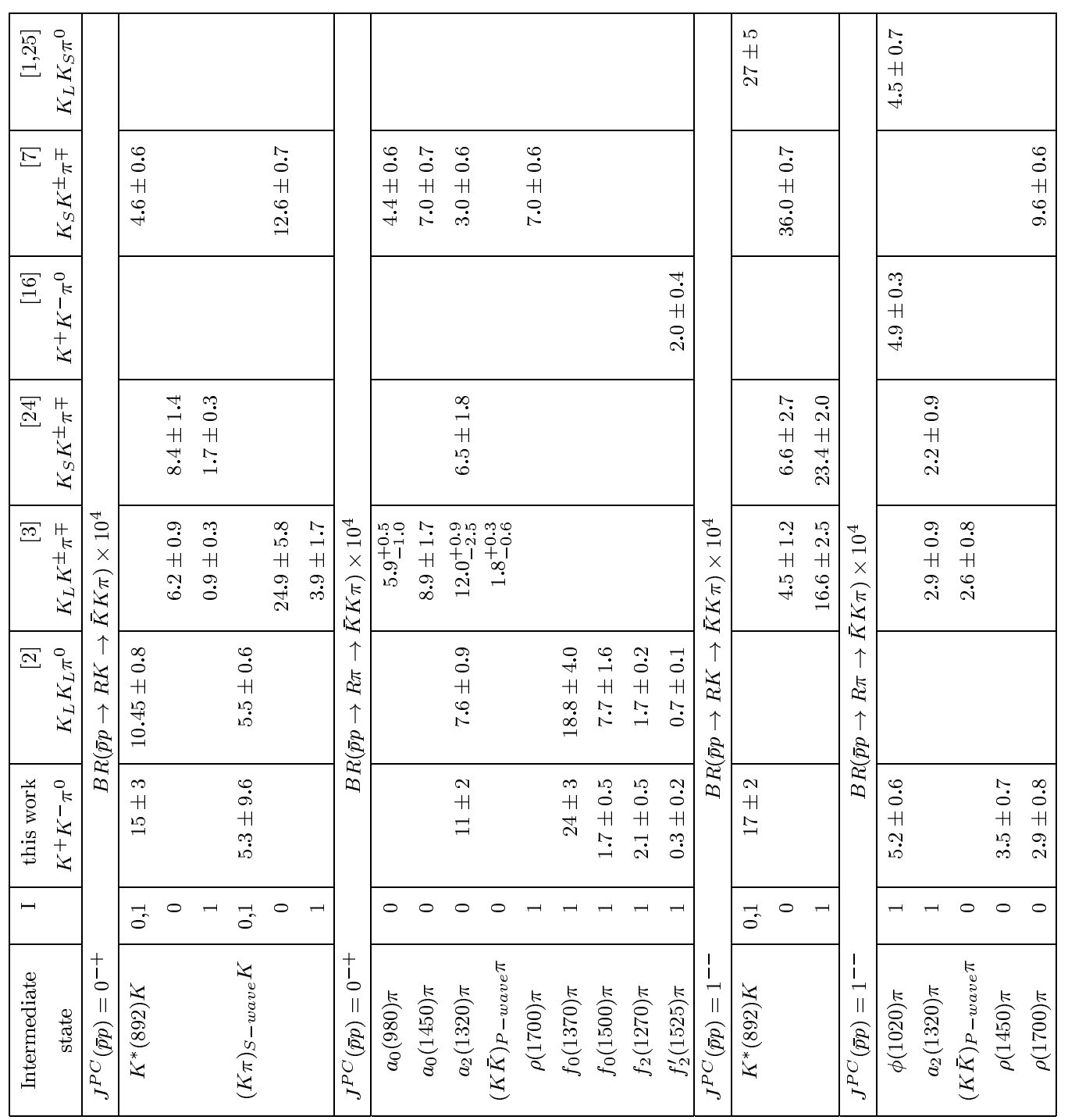

Table 2

Branching ratios for annihilation from a given $\bar{p} p$ initial state via various intermediate states into $\mathrm{K} \overline{\mathrm{K}} \pi$ final states; all $\mathrm{K} \overline{\mathrm{K}} \pi$ charge combinations are included. The values of previous results for the $\phi(1020) \pi$ branching ratio have been multiplied with the branching ratio $B R(\phi \rightarrow \mathrm{KK})=0.832$. 


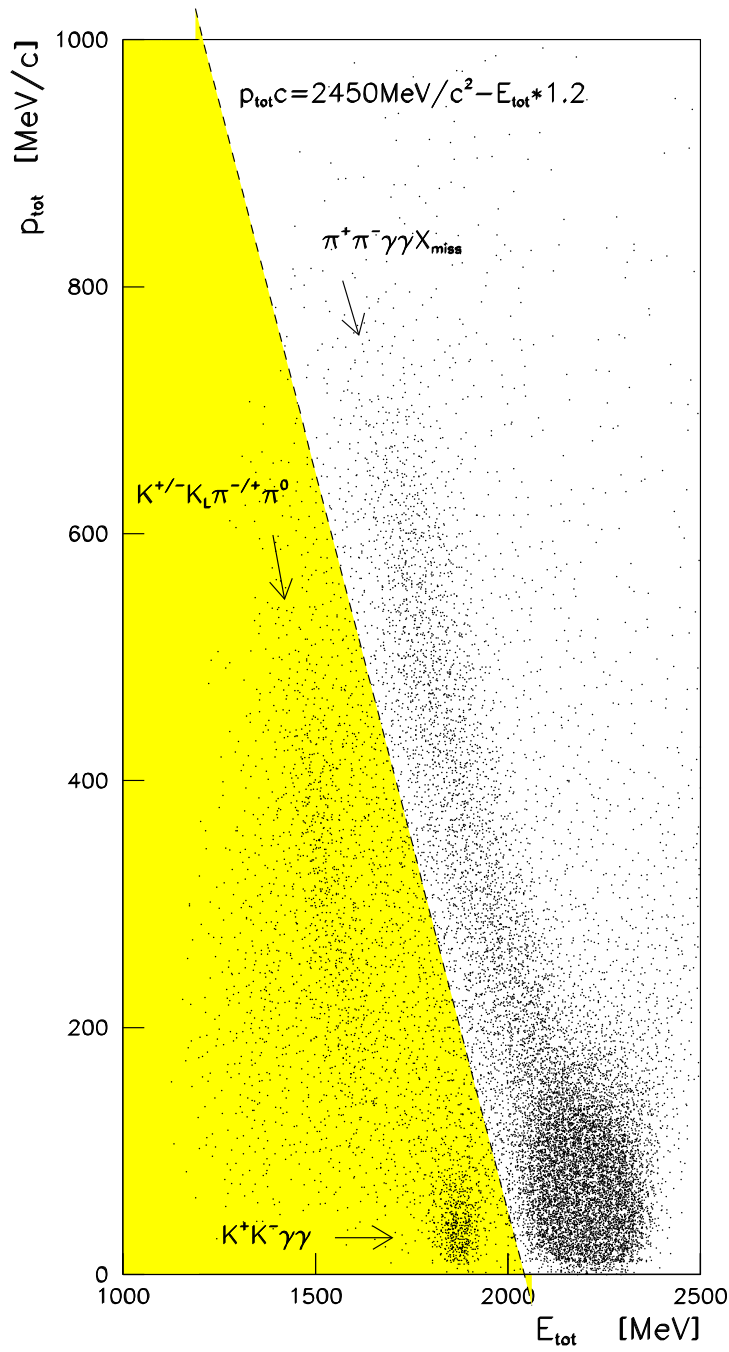

Fig. 1. Total momentum versus total energy in events with two tracks and two photons. If the two tracks are kaons, the total momentum in the event must be below the dashed line. The events from the $\mathrm{K}^{+} \mathrm{K}^{-} \gamma \gamma$ channel appear well separated from unwanted final states. 

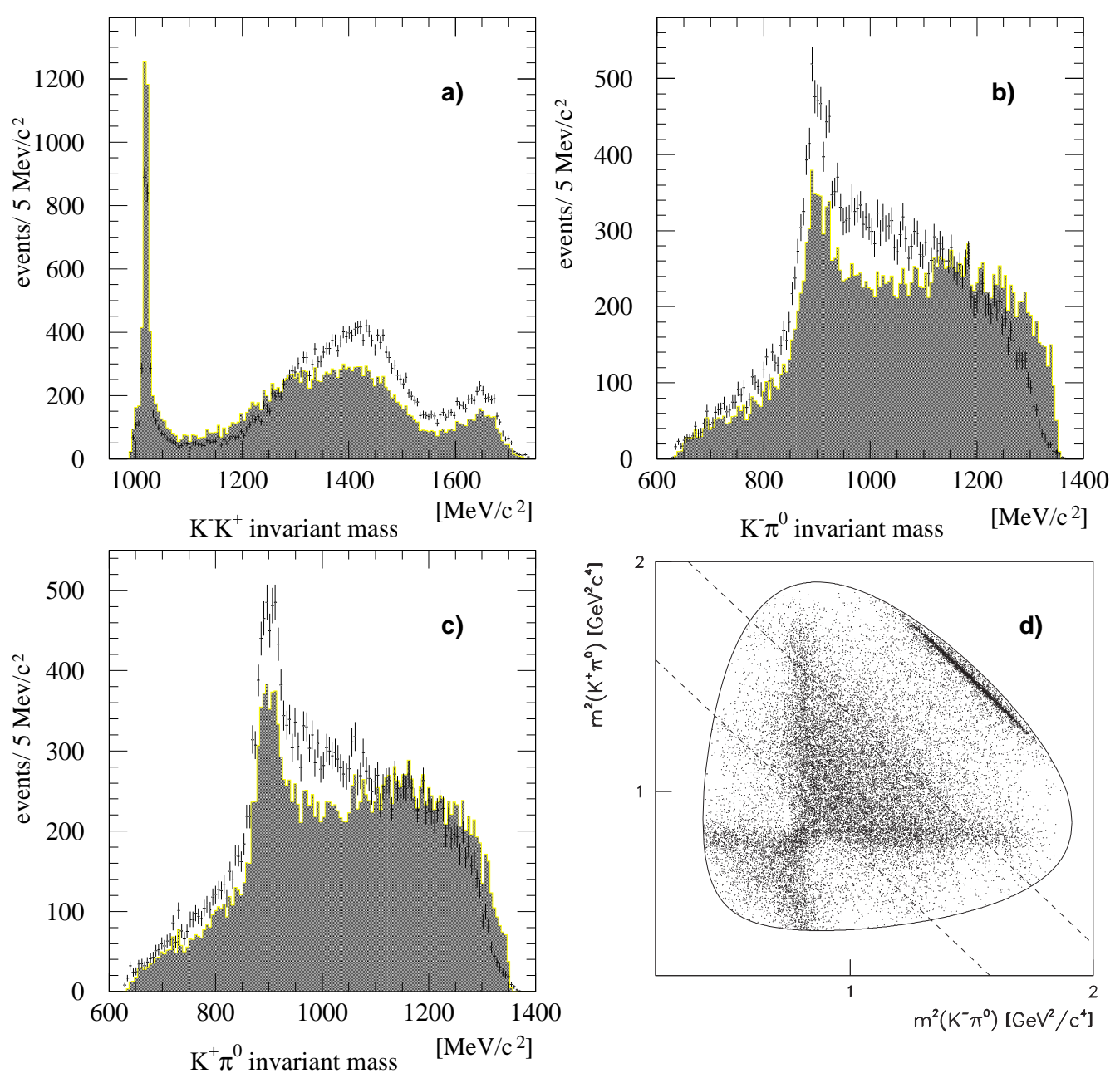

Fig. 2. The $\mathrm{K}^{+} \mathrm{K}^{-} \pi^{0}$ Dalitz plot and invariant mass distributions. Invariant mass distributions of a) $\mathrm{K}^{+} \mathrm{K}^{-}$; b) $\mathrm{K}^{-} \pi^{0}$; c) $\mathrm{K}^{+} \pi^{0}$. d) Dalitz plot with 27158 events. The dashed lines in $\mathrm{d}$ ) indicate invariant $\mathrm{K}^{+} \mathrm{K}^{-}$masses of $1.3 \mathrm{GeV} / c^{2}$ and $1.5 \mathrm{GeV} / c^{2}$. In $\mathrm{a}, \mathrm{b}, \mathrm{c})$ the distributions are also shown after all acceptance corrections were applied to the Dalitz plot (shaded area); they are scaled down by a factor 10, since the average reconstruction efficiency is $10 \%$. 

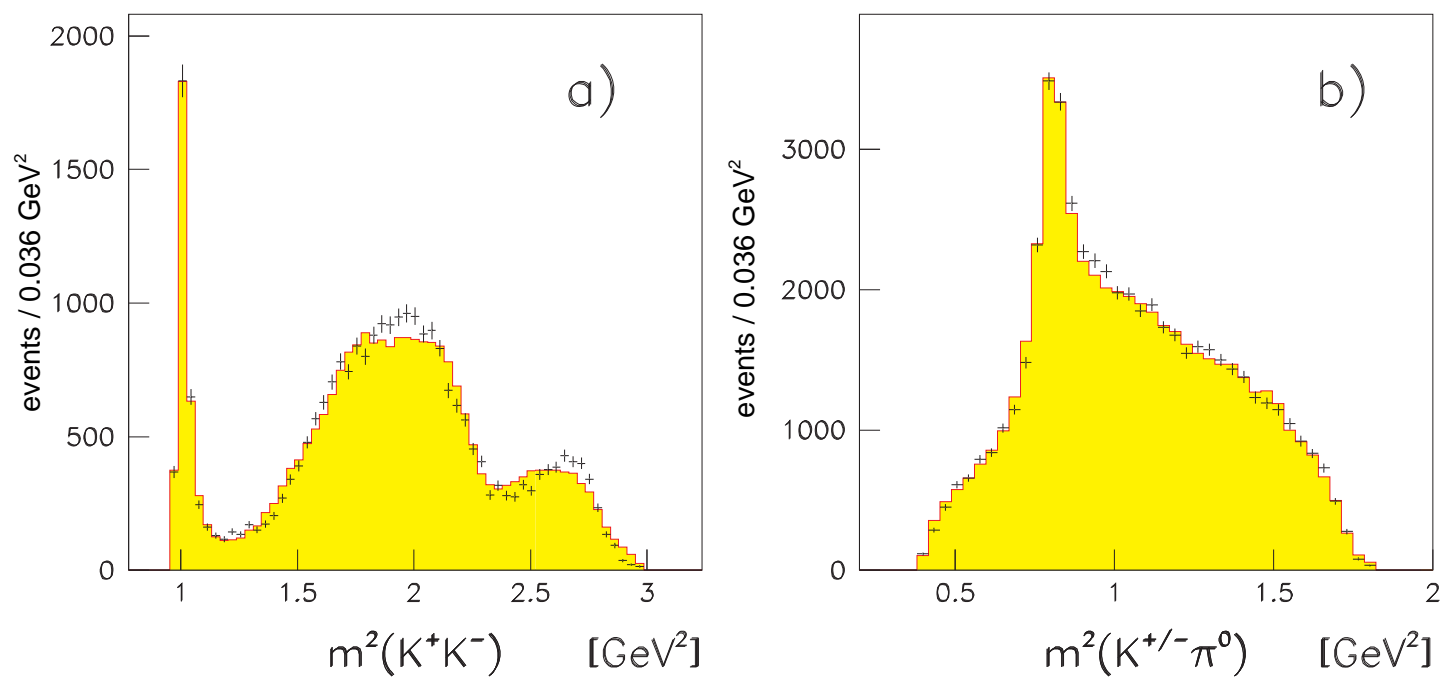

Fig. 3. Invariant mass distributions a) $\mathrm{K}^{+} \mathrm{K}^{-}$and b) $\mathrm{K}^{ \pm} \pi^{0}$. The experimental distributions are shown as points with error bars and the fitted distributions are shown as shaded histograms. The fit amplitudes are those of the basic hypothesis.
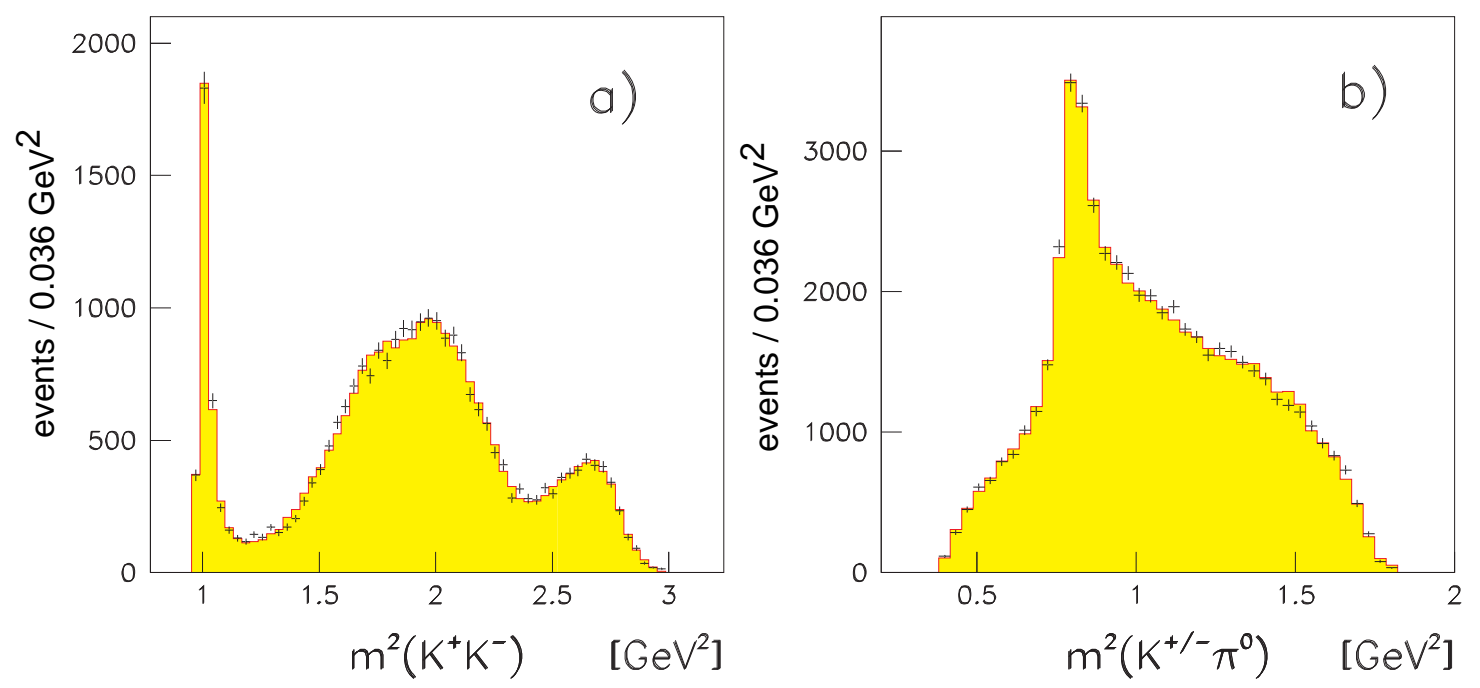

Fig. 4. Like the previous figure a) $\mathrm{K}^{+} \mathrm{K}^{-}$and b) $\mathrm{K}^{ \pm} \pi^{0}$ invariant mass distributions. The experimental distributions are shown as points with error bars and the fitted distributions are shown as shaded histograms. On top of the basic hypothesis, the amplitudes of this fit include a $(\mathrm{KK})$-P-wave with two poles. 